\title{
Novel mouse models shed light on role of omega-6/ omega-3 imbalance in chronic disease
}

\section{The link between two classes of essential fatty acids - omega-3 and omega-6 - and health has, until now, been unclear. This is partly because it has been difficult for researchers to define exactly what each does in the body. To address this problem, Dr Jing X. Kang of Massachusetts Genera Hospetically modified mice, each of which was able to produce defined types and quantities of the fatty acids. The work revelod thad the tissue/cellup ratio between omega-6 and omega-3 is crucial and of great significance in the development of chronic diseases such as cancer and diabetes. \\ $\mathrm{n}$ many fields of medical care,
treatments are now available th would have been impossible even despite these advances, chronic \\ diseases are on the rise. Around the world, the number of people living with serious conditions such as diabetes,
cascular disease and cancer is steadily and constantly increasing.}

\section{n-6/n-3 Imbalance}

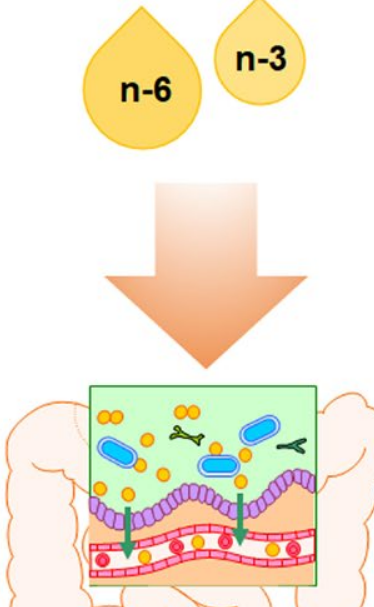

Gut Dysbiosis

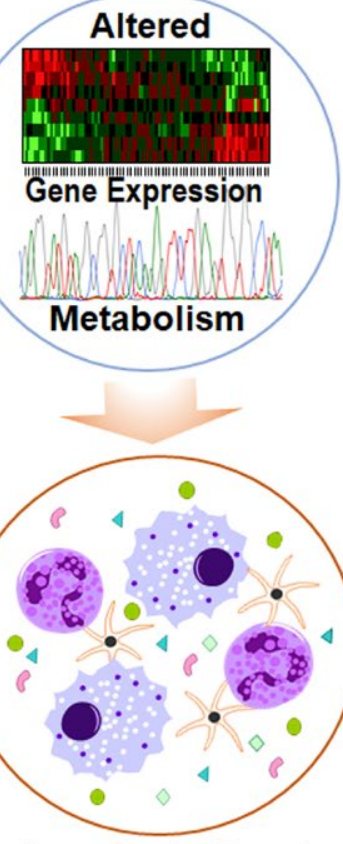

Low-Grade Chronic Inflammation
There are many possible explanations, ranging from genetics to lifestyle, for However, researc in chronic illness. that the growing prevalence of chronic diseases appears to coincide with na increase in omega- 6 fatty acids in the diet, and a corresponding decrease in omega- 3 fatty acids. This change is likely due to the shift in farming methods and a move towards processed foods in many parts of the world.

Both omega- 6 and omega- 3 are essential for health and must be obtained from food; these are the only

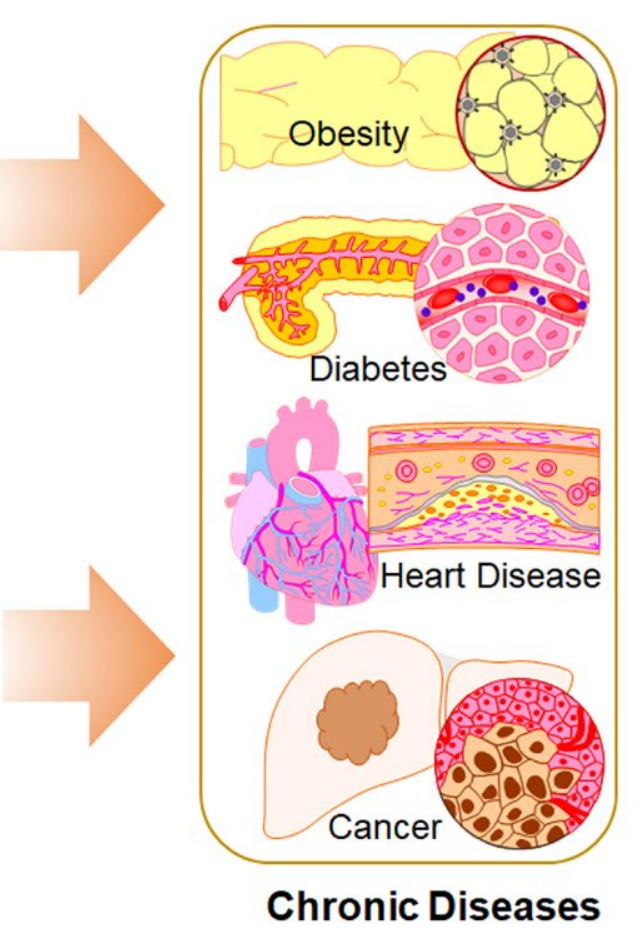

two fatty acids that humans cannot found in 'hears' foods like na-6, is 'nd regetably' oils, but is also comm in processed foods and snacks, and fatty or cured meat Omega-3 is also found in certain nuts and seeds, as well as fish like mackerel, salmon and sardines. These fatty acids play many vital roles in the body: they are essentia components of cell membrane and are involved in inflammatory processes and blood clotting, for example.

Some research has linked high levels of omega- 6 to chronic inflammation in the body. This could contribute to serious and long-term conditions like arthritis Confusingly, however, other studies have contradicted his clam, wh the resuls byg coung that on gab is not only safe cardiovascular health. Meanwhile, other researchers have found that omega-3 may be able to reduce inflammation. To unravel the true benefits and risks of these two essential fatty acids, a new approach is needed.

REVEALING THE FATTY ACID RATIO acids in health and disease have tended to focus on either omega-3 or omega- 6 . The relationship between the two fatty acids, and particularly the tissue/ cellular ratio of omega-6 to omega-3, has, untill now, been neglected. This is partly because it has proven difficult to de prinence of other nuthenty whidin the presence of other nutrients, which

To tackle this problem, Dr Jing X. Kang and his team at Massachusetts General Hospital and Harvard Medica School have developed unique strains of genetically modified mice. These levels of the ability to produce certain within the nom In previous and omega- 3 have been controlled 6 supplemented feed. The novel approach taken by Dr Kang and his colleagues allows the researchers to study the efects of varying ratios of the two fatty acids, whout the confounding factor of diet which has hind

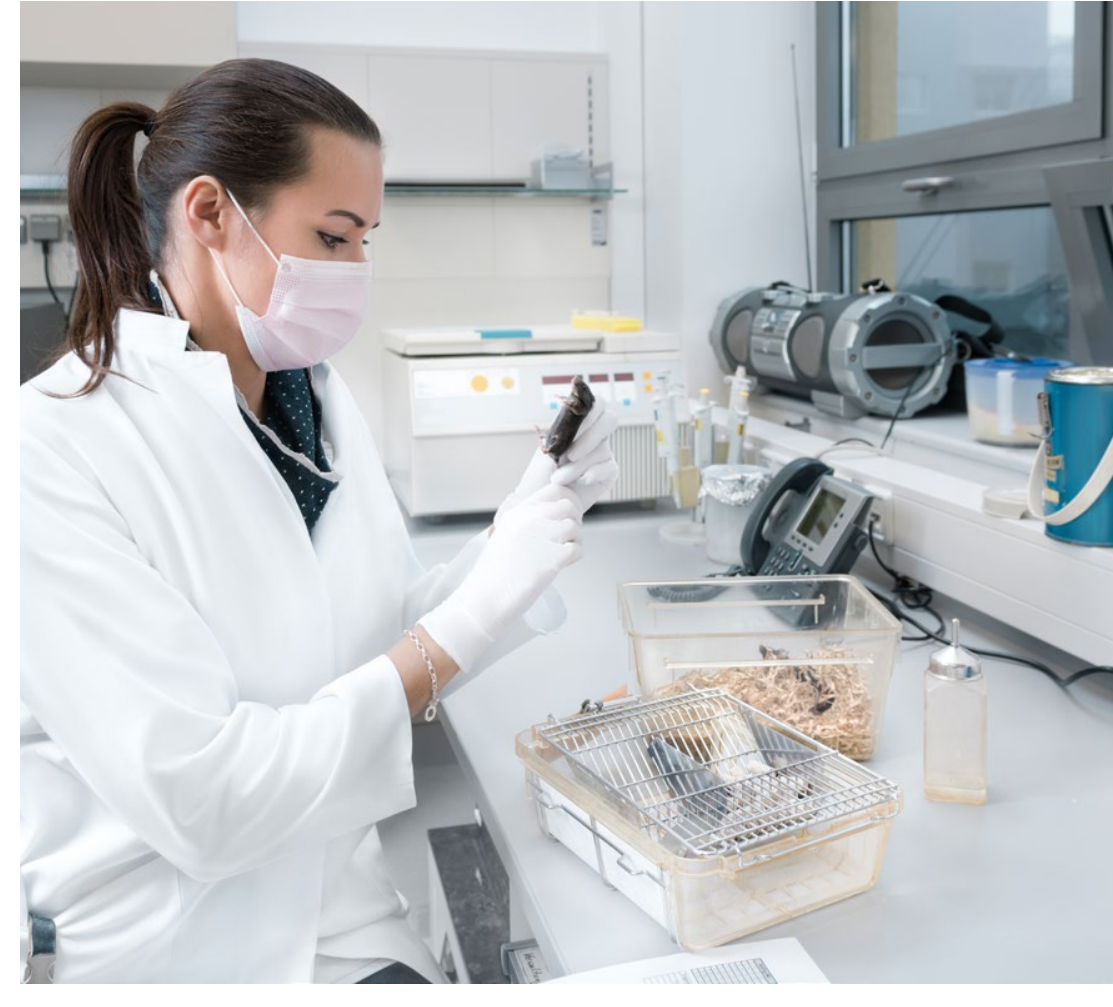

The research team has developp
levels of omega- 6 and omega- 3 .

In a recent project, Dr Kang used different types of mice to investigate the effects of the omega-6/omegaratio on metabolic conditions and chronic diseases. Four groups of mice were selected: wild-type, or 'normal', mice, which cannot synthesise omega strain the team called FAT- 1 , which strain the team called FAT-1, which

Researchers have noticed that the growing prevalence of chronic diseases appears to coincide with an increase in omega-6 fatty acids in the diet.

produces only omega- 6 ; and FAT1 +2 , which produces both omega- 6 and omega-3 fatty acids in a ratio close to $1: 1$. All of the mice were fed an identical diet.

In order to investigate the effects of different omega-6/omega-3 ratios, the research team used the transgenic techno Multi-omics refers to type techology. Multionics refers to a type data sets are consided, including the genome (i.e. all the genetic material (t) metabolome organism), and more.

OMEGA-6/OMEGA-3 RATIO INFLUENCES DISEAS The results showed what Dr Kang had The results showed what Dr Kang had omega-3 influences the development of chronic disease. The varying ratio of the two fatty acids seemed to affect the mice in a number of different ways: in their gut microbiota and in the product also dabelism, for example. The mice condition in their susceptibility to glucose intolerance obesily, fatyliver,

Of the four types of mice, the FAT-2 which synthesise omega- 6 only and 

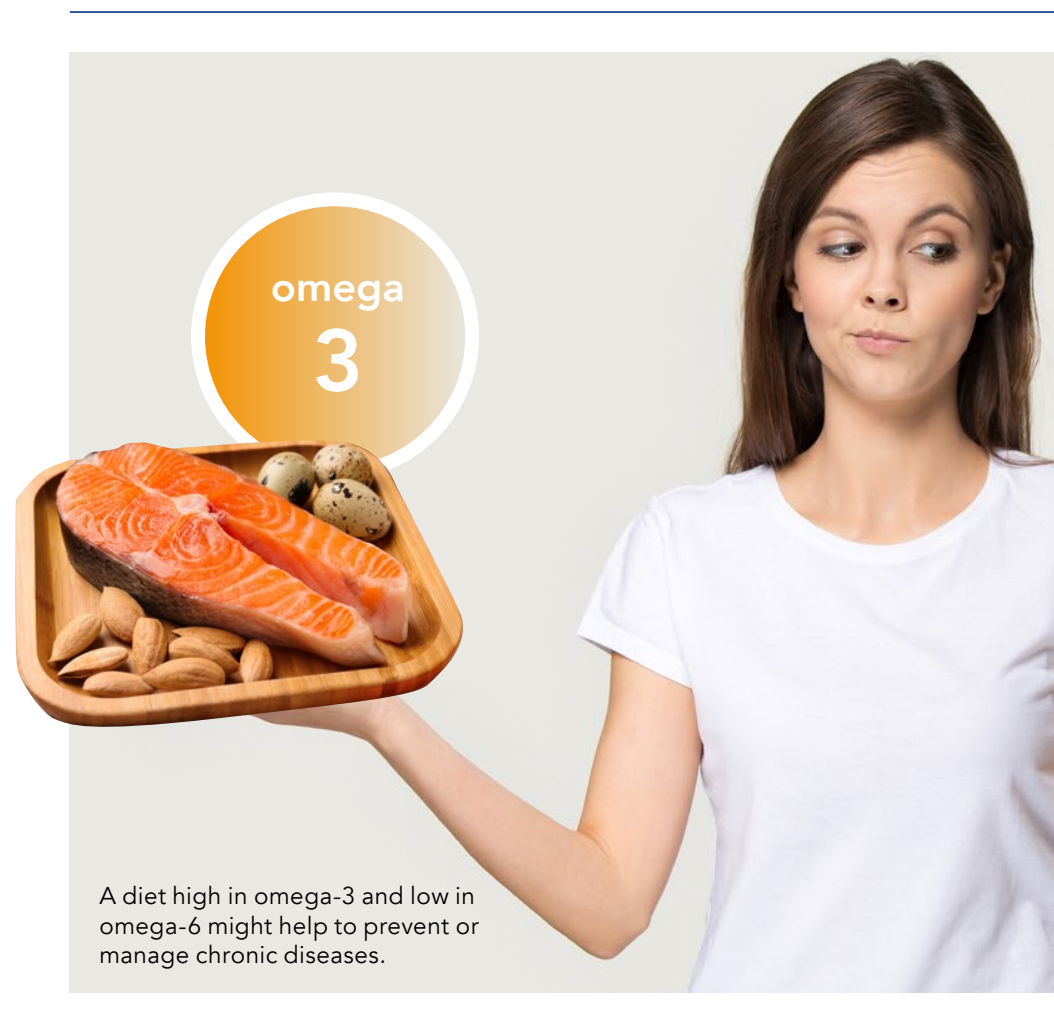

omega-3 - were the most likely to cancer. In fact, by the end of the study, most of the mice in this group had. developed liver cancer. In contrast. the FAT- 1 and FAT1 +2 mice were at far lower risk of these conditions. The latter two groups of mice had a balanced ratio of the two fatty acids, as their bodies were able to convert omega 6 to omega-3. Interestingly, the FAT-2 mice were also heavier, with a higher level of body fat, than the other three groups - despite all the mice receiving

Further analysis of the data revealed the development of long-term inflammation in the body. The FAT-2 mice also had a correspondingly low number of anti-inflammatory gut microbiota as a result of a high. omega-6/omega-3 ratio could play an important role in the development of

\section{IS OMEGA-6 RESPONSIBLE FOR} CHRONIC DISEASE? While previous research has suggested a link between omega- 6 and the development of chronic disease, the precise way in which this might happen results obtained by DrKang and his

The ratio of omega- 6 to omega-3 had profound effects on the gut microbiota of the mice.

had profound effects on the gut microbiota of the mice. There wer clear differences in the types of bacteria found in the FAT-2 mice when compared with the other three groups, and especially with the FAT- 1 and

FAT1+2 mice.

Many of the bacterial species found in the FAT-2 mice were pro-inflammatory in colleagues suggest a route via which the omega-6 to omega-3 ratio might directly influence chronic disease.

Dr Kang's research has shown that the two essential fatty acids, while similar in many ways, are not equal. In fact, the two substances appear to exert not
just different, but opposite influences in some chronic conditions. While omega-6 tends to promote While

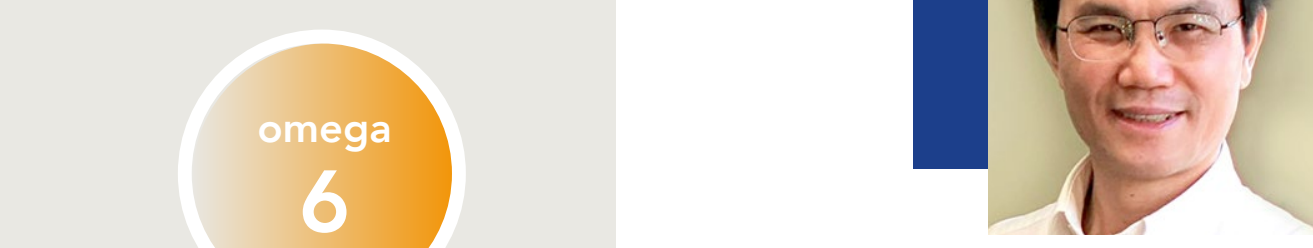

\section{Behind the Research}

Dr Jing $X$. Kang

E: jxkang@mgh.harvard.edu T: +1 6177268509 W: http://www.llmt-harvard.org

\section{Research Objectives}

Prof Kang is evaluating the impact of varying amounts of omega- 6 and omega- 3 fatty acids and their ratios on

\section{Detail}

Jing X. Kang

Laboratory for Lipid Medicine and Technology 149-13th Street, Room 4001

Boston, MA 02129, USA

Bio

Iflammation - which can, in turn, lead to other serious conditions - omega-3 tends to do the opposite. Overall, there is good evidence that excessive consumption of omega-6 is linked to poor health. To balance the ratio of essential fatty acids, an increase in a-3 is likely to be necessary.

PREVENTING CHRONIC DISEASE THROUGH DIETARY CHANGE The research conducted by Dr Kang and his team clearly suggests that a die high in omega- 3 and low in omega- 6 might help to prevent or manage many implication for public hes significant which often does not differentiate between the two essential fatty acid.

Research suggests that most people alive today have an omega- 6 to omega-3 ratio of around 10:1 or higher. To put this into context, for most of human history the ratio would have been close to 1:1. Given the everincreasing prevalence of diseases such as cancer, cliabetes and arthritis, most ofus are likely to encounter at least one of these conditions during our lifetime. Dr Kang's research strongly suggests that reducing omega- 6 in our diets, while boosting omega-3, could help to prevent these debilitating illnesses almost everyone, around the world. Medicine and Technology at Massachusetts General Hospital and Harvard Medical School. He is currently
Bio

X. Kang is the director of the Laboratory for Lipid the President of the International Society for Omega-3 Research. He is an expert in using genetically modified omega-6/omega-3 ratio on health and disease.

Funding

Fork

- Sansun Life Science Group (Hong Kong)

Collaborators

- Kanakaraju Kaliann

- Xing Yong

- Oian Pan

- Chih-Yu Chen

- Lei Hao

- Shanfu Xie

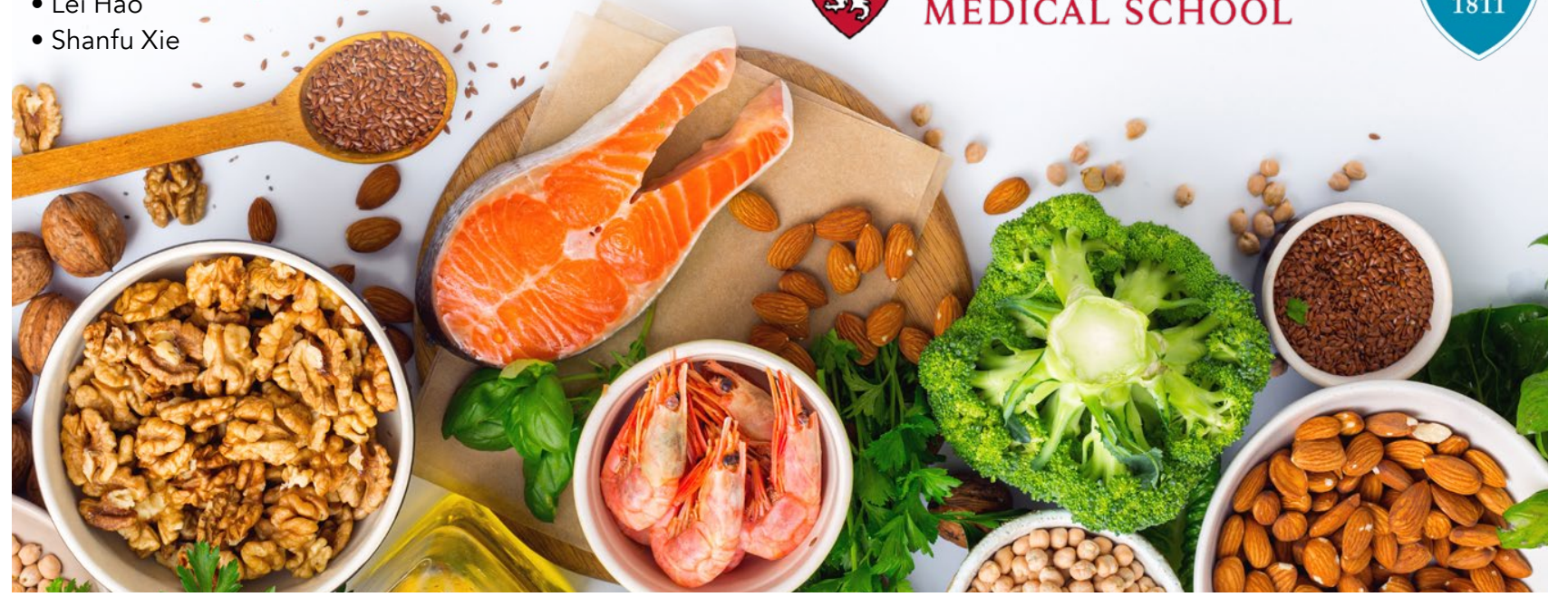
of FAT-1 transgenic mice have been published in top

\section{References}

Kaliannan, K., et al. (2019). Multi-omic analysis in transgenic mice implicates omega-6/omega-3 fatty acid imbalance as a risk factor for chronic disease. Communications Biology, 2, 276. Avaliable at:

\section{Personal Response}

Are there any other areas in which the transgenic mice used in this research might be useful?

II The creation of the FAT-1 mouse model has opened made it possible to study the authentic biological functions of omega- 6 and omega- 3 fatty acids without the confounding factors of diet, thereby overcoming the many hurdles of conventional dietary studies. Since has ben widely used 2004, the FAT-1 mouse model worldwide to study a variety of diseases (over 50 different health conditions) ranging from obesity to cancer. So far, more than 200 scientific papers based on the use journals, which have generated a vast pool of knowledge fatty acids and greatly increased our understanding of the importance of the omega-6/omega- 3 ratio in the development of chronic disease.

\section{HARVARD}

